\title{
Analysis of Conceptualization Patterns across Groups of People
}

Kano Glückstad, Fumiko ; Herlau, Tue; Schmidt, Mikkel N.; Mørup, Morten; Rzepka, Rafal ; Araki, Kenji

Document Version

Final published version

Published in:

Proceedings of 2013 Conference on Technologies and Applications of Artificial Intelligence,

Publication date:

2013

License

CC BY-NC-ND

Citation for published version (APA):

Kano Glückstad, F., Herlau, T., Schmidt, M. N., Mørup, M., Rzepka, R., \& Araki, K. (2013). Analysis of

Conceptualization Patterns across Groups of People. In Proceedings of 2013 Conference on Technologies and Applications of Artificial Intelligence, (pp. 349-354). IEEE.

Link to publication in CBS Research Portal

\section{General rights}

Copyright and moral rights for the publications made accessible in the public portal are retained by the authors and/or other copyright owners and it is a condition of accessing publications that users recognise and abide by the legal requirements associated with these rights.

Take down policy

If you believe that this document breaches copyright please contact us (research.lib@cbs.dk) providing details, and we will remove access to the work immediately and investigate your claim. 


\title{
Analysis of Conceptualization Patterns across Groups of People
}

\author{
Fumiko Kano Glückstad \\ Dept. of International Business Communication \\ Copenhagen Business School, CBS \\ Frederiksberg, Denmark \\ Email: fkg.ibc@cbs.dk
}

Tue Herlau, Mikkel N. Schmidt, Morten Mørup

Section for Cognitive Systems, DTU Compute

Technical University of Denmark, DTU

Kgs Lyngby, Denmark

Email: \{tuhe,mnsc,mmor\}@dtu.dk

\author{
Rafal Rzepka, Kenji Araki \\ Graduate School of Information Science and Technology \\ Hokkaido University \\ Sapporo, Japan \\ Email: $\{k a b u r a$, araki\}@media.eng.hokudai.ac.jp
}

\begin{abstract}
This paper analyzes patterns of conceptualizations possessed by different groups of subjects. The eventual goal of this work is to dynamically learn and structure semantic representations for groups of people sharing domain knowledge. In this paper, we conduct a survey for collecting data representing semantic representations of 34 subjects with different profiles in gender and educational background. The collected data is analyzed by an approach combining two extended versions of the Infinite Relational Model (Kemp et al. 2006) [1]: multiarray Infinite Relational Model (Mørup et al. 2010) [2] and normal Infinite Relational Model (Herlau et al. 2012) [3]. Results indicate that the employed approach not only localizes similar patterns of conceptualization within a group of subjects having a common profile, but also identifies differences in conceptualization across different subject groups.
\end{abstract}

Keywords-semantic representation; collective intelligence; Bayesian statistics; categorization; multi-array data analysis; psycholinguistics

\section{INTRODUCTION}

The globalization in the contemporary societies accelerated by the emergence of the Internet have brought about the inexorable rise in the use of English as a lingua franca in international communications. Whereas English is considered as an efficient means of communication, it generates a risk for leading to 'unwanted' misunderstandings and effects unconsciously influenced by non-native speakers' cultural and mental universe [4]. A cognitive scientist, Murphy [5] states that, in adult's second language acquisition, a newly encountered foreign object is aligned with one's priorknowledge, i.e. the taxonomic organization of categories that has been developed since childhood. This implies that, if the taxonomic organization of categories is deeply rooted in individual's background knowledge, such differences in conceptualization will affect our inference process in communication.

In our previous work [6], we investigated an approach for optimally identifying a common semantic structural grid in multiple concept-feature matrices, from which the individual semantic structures possessed by the respective subjects have been contrasted to each other. The obtained results indicated that the identified common grid effectively visualized the different feature association patterns possessed by four individuals. However, our previous work has been limited to the validation of the proposed approach, since the employed dataset publicly available by [7] has not disclosed the background profiles of each subject such as gender etc.

In this paper, we conduct an experiment for investigating conceptualization patterns of 34 subjects who undertook a feature-association test of 19 English words in the domain of clothing items. The collected data is analyzed by the approach combining multi-array Infinite Relational Model [2] and normal Infinite Relational Model (n-IRM) [3], which are the extended versions of the Infinite Relational Model (IRM) originally proposed by [1]. The multi-array IRM in parallel bi-clusters concept-feature relations across multiple matrices and identifies a common semantic structural grid across multiple subjects. This means that the identified concept- and feature clusters that are partitioned according to the common grid are consistent across subjects, while the interactions between concepts and features are subject specific. Through this identified grid, both similar and different patterns of concept-feature relations indicated by the respective subjects are inspected. For further looking into patterns of the interactions, the n-IRM is applied to density values of the interactions between a concept- and a feature cluster across 34 subjects, obtained from the multi-array IRM.

Section 2 explains the experimental setting for the data collection, followed by the models employed for the data analysis in Section 3. Section 4 analyzes the collected data followed by discussions and conclusion in Sections 5 and 6 .

\section{DATA COLLECTION}

In the previous work [6], the publicly available dataset prepared by [7] was used for validating the proposed ap- 
proach. The dataset used in the previous work is comprised of matrices of four subjects. Each matrix consists of 29 concepts of clothing items, e.g. blouse, pants, dress, and their 258 features, e.g. protects against the cold, made of silk, gives a business like impression. For every feature-concept pair, the four subjects respectively assigned a binary value 1 , if a feature is applicable to the concept in question, otherwise 0 was assigned in [7]. Based on the IRM analysis [6] of De Deyne's [7] dataset, we selected 19 clothing items and 74 features that influenced the formation of clusters, and used these as the basis of our experiment in the present work.

The experiment employs 20 students (10 male, 10 female) studying computer science at Hokkaido University and 14 students (7 male, 7 female) studying English communication under native English speakers at Tokai University in Japan. All subjects are native Japanese speakers. Among these, three subjects are advanced English speakers who have experiences in living foreign countries. Each subject is asked to answer three types of on-line questionnaires: i) a picturenaming test where the subjects have to select one suitable English word (among the 19 English words) corresponding to images of clothing items displayed on the screen, ii) a categorization test where subjects are asked to freely categorize the 19 English words, and iii) a feature-association test where subjects have to select as many features as possible (among 74 features) related to each English word. In this paper, the data collected by the questionnaire iii) is analyzed. The collected data consists of concept-feature matrices of 34 subjects where binary value 1 is assigned when a subject considers a feature is applicable to a specific English word, and 0 when a feature is inapplicable.

\section{METHOD}

\section{A. Infinite Relational Model}

The approach employed in the present work combines multi-array Infinite Relational Model (multi-array IRM) [2] and normal Infinite Relational Model (n-IRM) [3], which are the extended versions of the Infinite Relational Model (IRM) originally proposed by [1]. The present work employs the simple model analyzing a dataset of two modes $T_{1}$ and $T_{2}$ with a two-place relation $R: T_{1} \times T_{2} \rightarrow\{0,1\}$, where $T_{1}$ and $T_{2}$ respectively correspond to the English words (concepts) and their features. For parallel analysis of multiple matrices, multi- array IRM [2] is employed to the binary matrices.

The IRM [1] generates clusters based on an induction process called the Chinese Restaurant Process (CRP) [8]. The CRP starts a partition by creating a single cluster with only one object, and subsequently adds other objects to the partition by letting the $i^{\text {th }}$ object either select a new cluster with probability $\frac{\gamma}{i-1+\gamma}$, or an existing cluster $a$ with probability $\frac{n_{a}}{i-1+\gamma}$. Here, $n_{a}$ refers to the number of objects already assigned to cluster $a$, and $\gamma>0$ is a parameter [1].
The clusterings of the two modes $T_{1}$ and $T_{2}$ are respectively defined as $\mathbf{z}^{(1)}$ and $\mathbf{z}^{(2)}$, which means that, in the first- and the second modes of the following generative model, concepts and features are partitioned into $\mathbf{z}^{(1)}$ and $\mathbf{z}^{(2)}$ clusters. The third mode defines how concept clusters relate to feature clusters for the $s^{t h}$ subject by $\eta_{a b}^{(s)}$. This is computed by $p\left(R_{i j}^{(s)}=1 \mid \mathbf{z}^{(1)}, \mathbf{z}^{(2)}, \boldsymbol{\eta}^{(s)}\right)=\eta_{z_{i}^{(1)} z_{j}^{(2)}}^{(s)}$. Binary relations for the $s^{t h}$ subject between concept $i$ and feature $j$, i.e. $R_{i j}^{(s)}$, are thereby drawn based on the extracted clusters and their subject specific interactions.

$$
\begin{aligned}
\mathbf{z}^{(1)} & \sim \operatorname{CRP}\left(\gamma^{(1)}\right) & & \text { first mode, } \\
\mathbf{z}^{(2)} & \sim \operatorname{CRP}\left(\gamma^{(2)}\right) & & \text { second mode, } \\
\eta_{a b}^{(s)} & \sim \operatorname{Beta}\left(\beta_{0}^{+}, \beta_{0}^{-}\right) & & \text {interactions, } \\
R_{i j}^{(s)} & \sim \operatorname{Bernoulli}\left(\eta_{z_{i}^{(1)} z_{j}^{(2)}}^{(s)}\right) & & \text { links. }
\end{aligned}
$$

In the above model, prior parameters are set as $\beta_{0}^{ \pm}=1$, and $\gamma^{(t)}=\log \left(J_{t}\right)$ where $J_{t}$ is the number of concepts in each mode, i.e. $t \in\{1,2\}$. Inference in the model is, as described in [2], performed by Gibbs sampling combined with a split-merge Metropolis Hastings framework. The solutions presented in Section 4 are based on the single realization with highest likelihood.

\section{B. Normal Infinite Relational Model}

The normal IRM (n-IRM) [3] that is an extended model of the IRM employs normal distribution as likelihood function and normal-inverse gamma priors instead of the Bernoulli likelihood and beta priors. The n-IRM draws on a Bayesian generative model that partitions each mode of matrix $W_{i j}$ into clusters again defined by $z^{(1)}$ and $z^{(2)}$ respectively. The interaction between the extracted groups of each mode is parameterized by a mean intensity $m_{a b}^{(s)}$ and precision $\lambda_{a b}=\sigma_{a b}^{-2}$, which are given by a Normal-Gamma prior in the the following generative model. The details of the entire generative process is described in [3].

$$
\begin{aligned}
\mathbf{z}^{(1)} & \sim \operatorname{CRP}\left(\gamma^{(1)}\right) & & \text { first mode, } \\
\mathbf{z}^{(2)} & \sim \operatorname{CRP}\left(\gamma^{(2)}\right) & & \text { second mode, } \\
\lambda_{a b} & \sim \operatorname{Gamma}\left(\alpha_{0}, \text { rate }=\beta_{0}\right) & & \text { precision, } \\
m_{a b} & \sim \operatorname{Normal}\left(m_{0},\left(\kappa_{0} \lambda_{a b}\right)^{-1}\right) & & \text { mean, } \\
R_{i j} & \sim \operatorname{Normal}\left(m_{z_{i}^{(1)} z_{j}^{(2)}}, \lambda_{z_{i}^{(1)} z_{j}^{(2)}}\right) & & \text { links. }
\end{aligned}
$$

In the present work, the n-IRM is applied to eta-values $\eta_{a b}$ which is the highest likelihood of relations between concept- and feature clusters computed by the multi-array IRM. The obtained 34 multi-array $\eta_{a b}$ matrices ( 4 concept clusters x 13 feature clusters) is reorganized in a way that a $\eta_{a b}$ value matrix of $T_{1}$ and $T_{2}$ respectively correspond to the 4 concept clusters $(C K 1-C K 4)$ and 34 subjects, i.e, the 
$\eta_{a b}$ value matrix (4CKs x 34 subjects) is created for each feature cluster obtained from the multi- array IRM. Thus the n-IRM is independently applied to these thirteen $\eta_{a b}$ value matrices. The prior values for the n-IRM was set to $\kappa_{0}=1$, $\alpha_{0}=15$ and $\beta_{0}^{-1}=1$ to reflect the scale of the $\eta$ values.

Inference is performed by gibbs sampling as in [2]. The gibbs sampler is run for 1000 iterations and the first 500 iterations is discarded as burnin. For quantifying stabilities of the obtained concept- and feature clusters, a commonly used measure called Normalized Mutual Information (NMI) [9] is employed. The NMI indicates a number between 0 and 1 such that 1 describes identical assignments to clusters.

\section{DATA ANALYSIS}

Fig. 1 overviews semantic representations of 34 subjects analyzed from several dimensions. The upper-left plot depicts the multi-array IRM results, which illustrates an optimal common semantic structural grid that partitions the $T_{1}$ mode (19 English words) and the $T_{2}$ mode (74 features) into $4 \times 13$ clusters across all 34 subjects. The stabilities of the obtained clusters (NMI scores) are listed at the left-upper part of Fig. 1. The 19 English words are reasonably categorized in a way that the obtained concept clusters CK1-4 respectively represent themes, i.e., CK1: casual items (pullover, top, sweater, T-shirt, pants, jeans, shirt), CK2: items for female (scarf, blouse, skirt, dress), CK3: underwear items (underpants, panties, bra, shorts), and CK4: formal items (tie, coat, jacket, suits). Members of the obtained 13 feature clusters are listed in Fig. 2. Here, for each feature cluster, the obtained $\eta_{a b}$ value matrices reorganized in Section 2 are further sorted by the n-IRM. The 13 plots of the middle column in Fig. 2 depict degrees of relations ( $\eta_{a b}$ values) between the concept clusters ( $C K 1$ $C K 4$ ) and a specific feature cluster answered by the 34 subjects, which are sorted based on the distributions of mean values and standard deviations (the square root of the precision) explained in Section 3. The gray scale in the plots indicates that higher $\eta_{a b}$ values close to 1 gets darker gray.

The plots in Fig. 2 indicate how each feature cluster influences the formation of concept clusters depending on different groups of subjects. For example, feature clusters 2 and 5 show stronger relations with $C K 4$ : formal items and $C K 3$ : underwear items, respectively. This indicates that the majority of subjects consider that feature clusters 2 and 5 strongly influence the formation of $C K 4$ and $C K 3$. Contrary, feature clusters 6 and 7 are rather subject dependent. To be more concrete, for feature cluster 6 consisting of (thin, light and airy, comfortable, soft, colorful), the subjects belonging to subject cluster 2 (SK2) indicate stronger relations with $C K 2$ and $C K 3$. Moreover, the table shown in the right column indicates that $S K 2$ for the feature cluster 6 is a female dominant cluster. By studying the plots and tables in Fig. 2, it is generalized that the subject clusters having darker gray colors are dominated by female subjects. This implies that female subjects tend to have stronger associatiation with each English word.

The results shown in Fig. 2 indicate that the association patterns of some feature clusters are subject dependent and some patterns seem to be dependent on the profiles of the subjects. Accordingly, we further analyzed how the conceptfeature matrices generated by a specific group of subjects are partitioned through an optimal common grid in Fig. 1. The 34 subjects are first divided into two groups from two dimensions, gender (male, female) and educational background (humanities and natural sciences), and the matrices of each group has been analyzed by the multi-array IRM. It identifies a common grid that partitions the matrices into concept- and feature clusters for each group of subjects. The number of clusters obtained and the distributions of cluster members are slightly different in the four groups in Fig. 1. For example, the obtained concept clusters for the two groups representing humanities and natural sciences are almost identical. However, the English word shorts is categorized as underwear items (CK2) among subjects in natural sciences and as casual items $(C K l)$ among subjects in humanities. The male subjects categorized panties, bra, skirt, shorts as one cluster distinguished from underpants (CK5), while the female subjects categorized underpants, panties, bra, shorts (CK3) as one cluster and skirt is included in another cluster representing items for females (CK2). Another point is that the subjects representing female as well as natural sciences respectively divided features into larger number of clusters. Stabilities (NMI scores) of the concept clusters are approximately 0.9 and above, which indicate that the obtained clusters are fairly reliable. The average NMI scores are particularly higher in humanities group, 1.0, and female group, 0.9792 .

These group of subjects are further divided into more specific four groups: 'male humanities', 'female humanities', 'male natural sciences' and 'female natural sciences'. Fig. 1 depicts individual conceptualizations represented via a common grid identified for these specific subject groups. Although individual differences are identified within each group of subjects in the plots in Fig. 1, these plots show some similar distribution patterns of concept-feature relations (dots) within each group of subjects. The clustering result shows that the concept clusters obtained by the female subjects both in humanities and natural sciences are exactly the same, although the number of feature clusters obtained by the subjects in 'female natural sciences' are larger. The average NMI scores for both 'female humanities' and 'female natural sciences' are high, i.e. above 0.9 and 0.8 for the concept- and the feature clusters, respectively. Contrary, the subjects in 'male humanities' categorized more concept clusters and fewer feature clusters, while the subjects in 'male natural sciences' categorized fewer concept clusters and more feature clusters. The average NMI scores for the concept clusters in the both male groups are substantially 

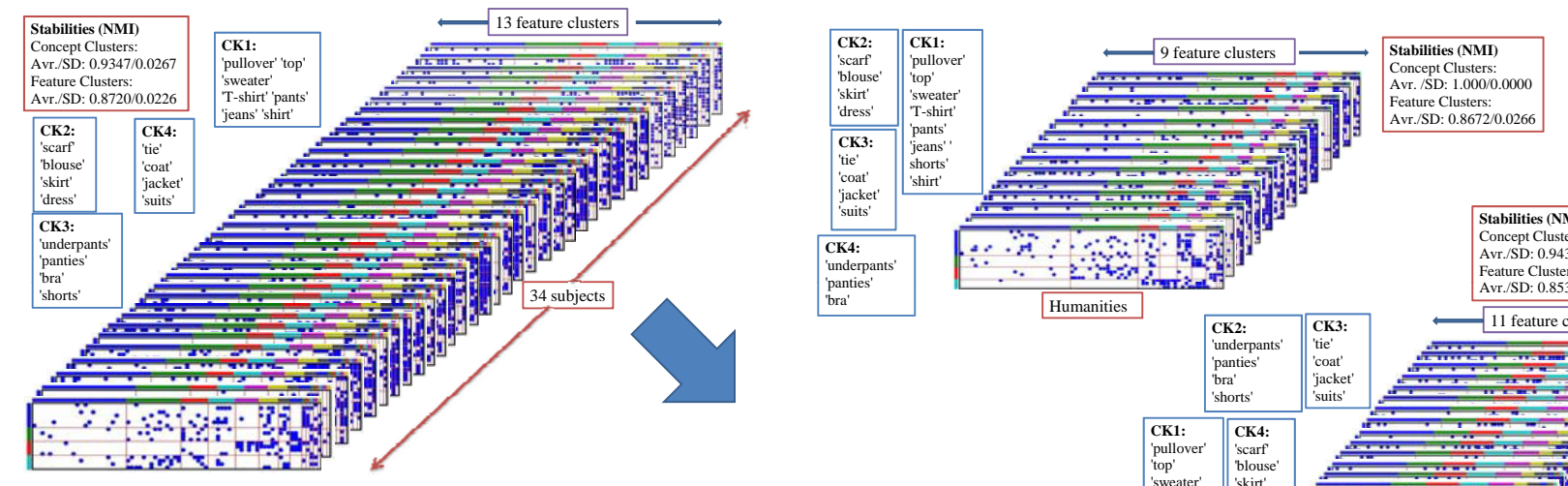

$.000 / 0.0000$ \begin{tabular}{|l|}
\hline Stabilities (NMI) \\
Concept Clusters: \\
Avr./SD: $0.9430 / 0.0246$ \\
Feature Clusters: \\
Avr./SD: $0.8530 / 0.0142$ \\
\hline
\end{tabular} 11 feature clusters
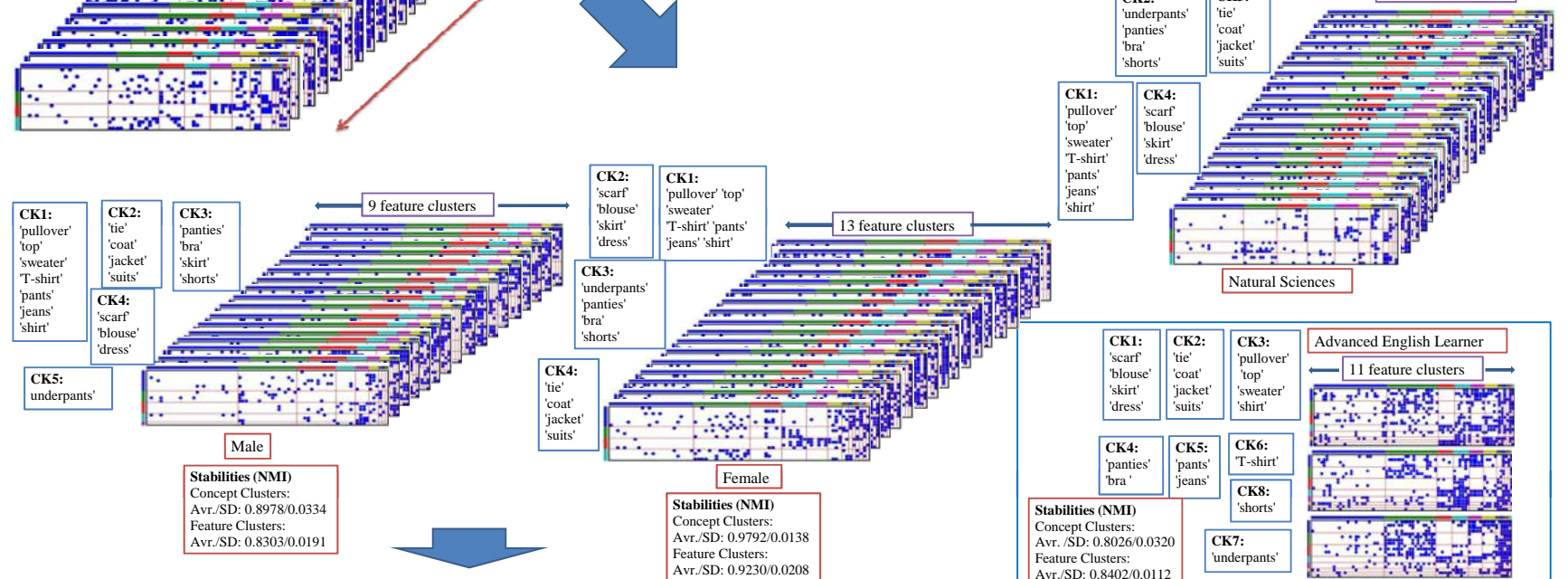

Natural Sciences
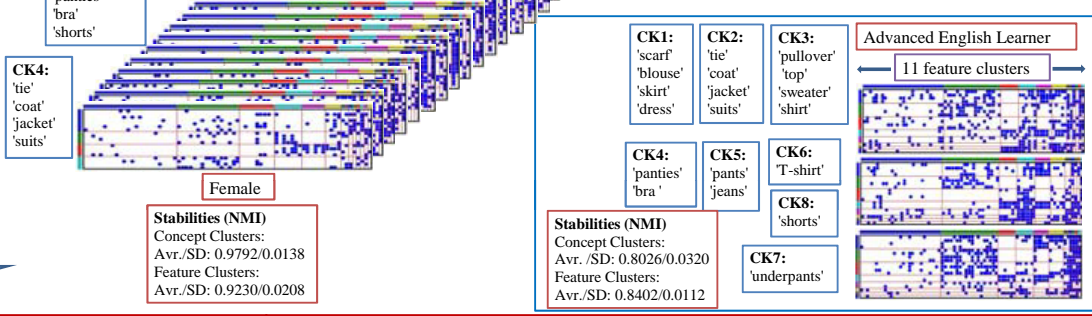

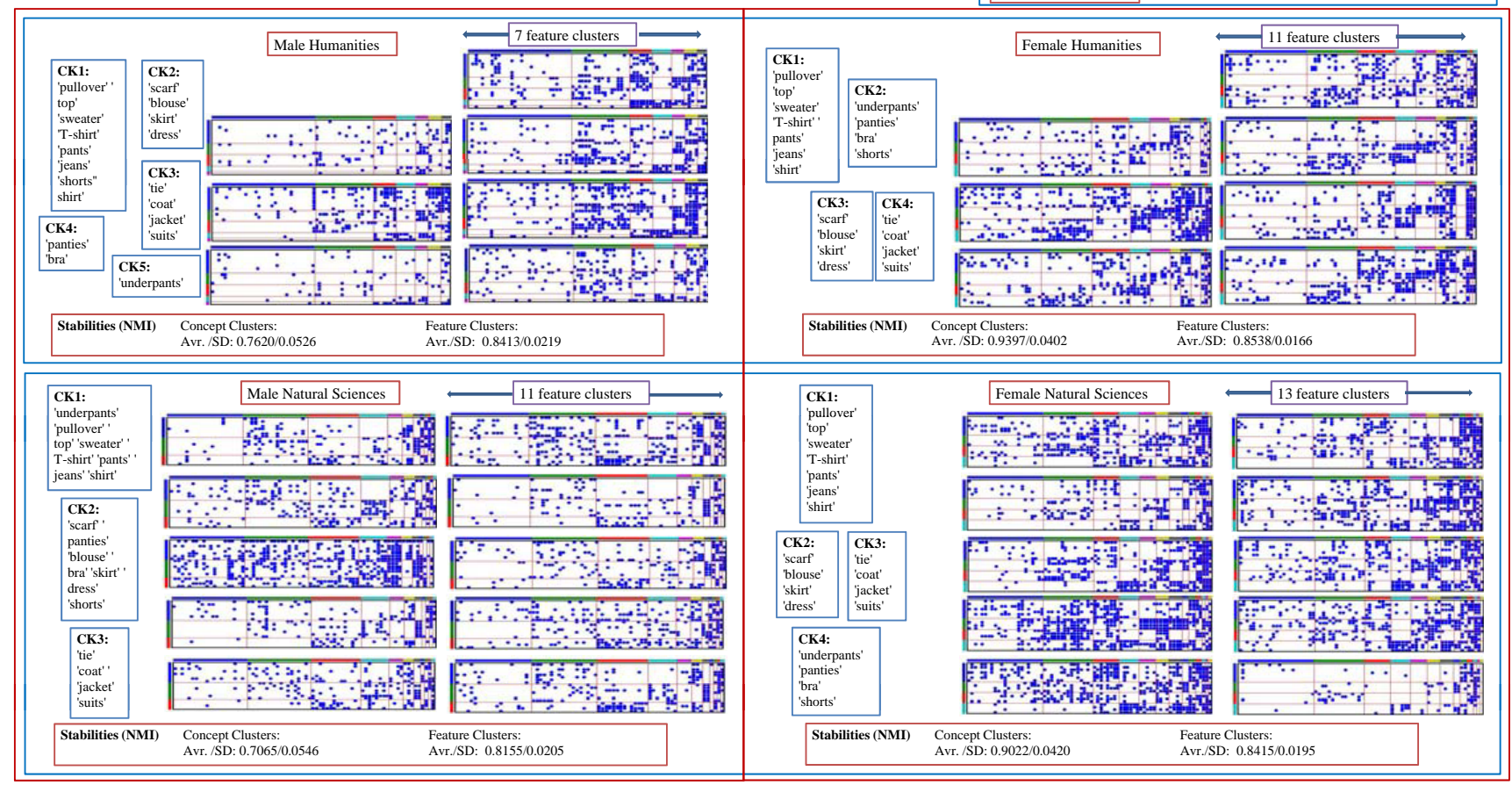

Figure 1. Multi-array analysis based on different groups of subjects

lower than the ones in the female groups.

\section{DisCUSSION AND FUtURE PERSPECTIVES}

The results presented in the previous section indicate several interesting phenomena. A possible interpretations of the results are as follows. The female subjects are more familiar with the clothing items expressed in English, and therefore have clearer feature associations to each concept. It seems that the subjects in natural sciences are logically 
Feature cluster 1:

'has/have short sleeves"' "'not sexy"' '"worn in Summer"

"consists of different pieces"' "'worn by young people"' '"is/are loose"

"made of cotton"' '"made of leather"' '"worn with jeans"' "'not expensive "'short'" 'made of denim"' "'made of wool"' '"covers both the upper- and bottom part of your body" ' "thick"' "tight"' "looks sporty"' '"used indoors" used on vacation"' '"'worn by elderly"' "uncomfortable"' "shrinks when washed in a washing machine"' '"used as accessory"' "need to be ironed"'

Feature cluster 2:

"'costs a lot of money"' "'gives a businesslike impression"' '"has/have a button"' "'has/have a collar"' "'can be a status symbol"' '"has/have long sleeves "' "'or formal occasions " is/are long " "Used outdoors" "worn in "worn by men"

Feature cluster 3:

"'beautiful"' '"made of silk"' '"fun to wear"' '"given as a present"' '"chic"' "'"'comes in very handy"' "'plain-colour'" '"popular"' "'worn as leisure time clothes"' "'for informal occasions"' '"worn in Spring or Autumn"' "'worn often"' 'used to look nicer"' '"worn at parties"'

Feature cluster 4:

'"comes in very handy"' '"plain-colour"' "'popular"' '"worn as leisure time clothes"' '"for informal occasions"' '"worn in Spring or Autumn"' "worn often"

Feature cluster 5:

"worn underneath other clothes"' '"worn every day"' '"is/are hygienic"' ' "is/are underwear"' "'worn on the bottom part of your body"

"prevents being naked"

\section{Feature cluster 6:}

"'thin"' '"light and airy"' "'comfortable"' '"soft"' "'colourful"

Feature cluster 7:

"worn on the upper part of your body"' '"for all ages"'

worn by both men and women"

Feature cluster 8: "'feminine"' '"worn by women"' '"sexy"'

Feature cluster 9: "'everybody has it"

Feature cluster 10: "'for all seasons"

Feature cluster 11: "'fashionable'

Feature cluster 12: 'has/have different patterns or shapes"

Feature cluster 13: '"can go into the washing-machine"'
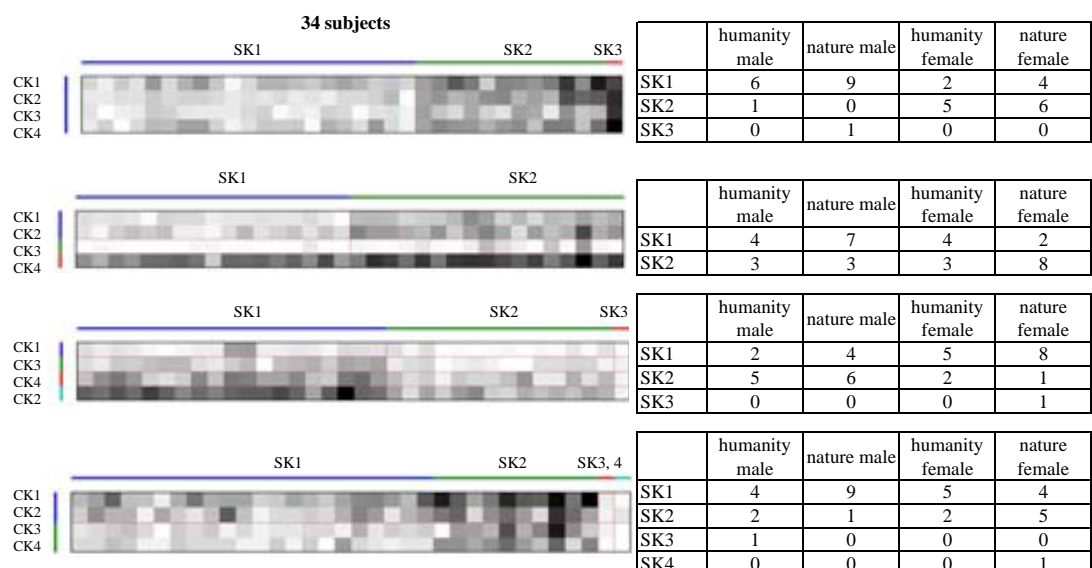

\begin{tabular}{|l|c|c|c|c|}
\hline & $\begin{array}{c}\text { humanity } \\
\text { male }\end{array}$ & nature male & $\begin{array}{c}\text { humanity } \\
\text { female }\end{array}$ & $\begin{array}{c}\text { nature } \\
\text { female }\end{array}$ \\
\hline SK1 & 4 & 7 & 4 & 2 \\
\hline SK2 & 3 & 3 & 3 & 8 \\
\hline
\end{tabular}

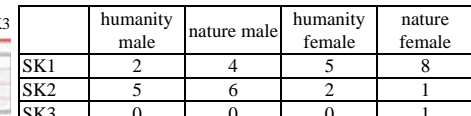

SK1

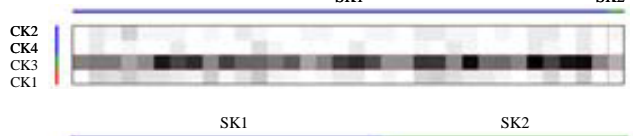

\begin{tabular}{|l|c|c|c|c|}
\hline & $\begin{array}{c}\text { humanity } \\
\text { male }\end{array}$ & nature male & $\begin{array}{c}\text { humanity } \\
\text { female }\end{array}$ & $\begin{array}{c}\text { nature } \\
\text { female }\end{array}$ \\
\hline SK1 & 4 & 9 & 5 & 4 \\
\hline SK2 & 2 & 1 & 2 & 5 \\
\hline SK3 & 1 & 0 & 0 & 0 \\
\hline SK4 & 0 & 0 & 0 & 1 \\
\hline
\end{tabular}

\begin{tabular}{|l|c|c|c|c|}
\hline & $\begin{array}{c}\text { humanity } \\
\text { male }\end{array}$ & nature male & $\begin{array}{c}\text { humanity } \\
\text { female }\end{array}$ & $\begin{array}{c}\text { nature } \\
\text { female }\end{array}$ \\
\hline SK1 & 6 & 10 & 7 & 10 \\
\hline SK2 & 1 & 0 & 0 & 0 \\
\hline
\end{tabular}
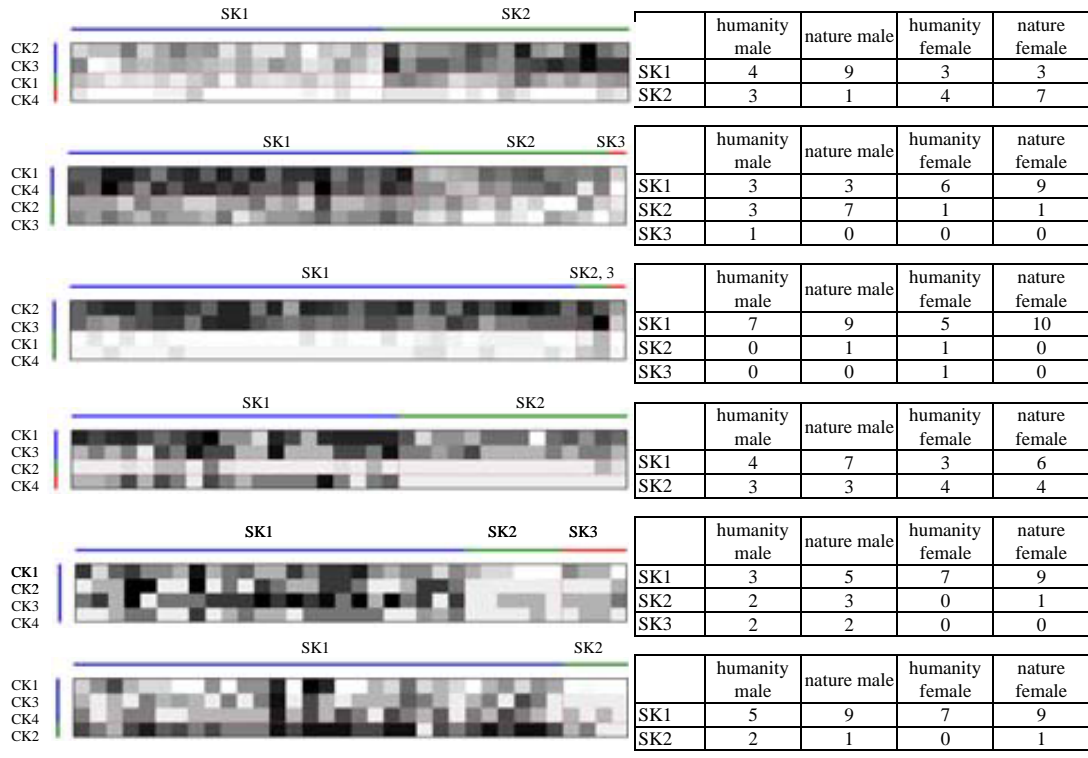

\begin{tabular}{|l|c|c|c|c|}
\hline & $\begin{array}{c}\text { humanity } \\
\text { male }\end{array}$ & nature male & $\begin{array}{c}\text { humanity } \\
\text { female }\end{array}$ & $\begin{array}{c}\text { nature } \\
\text { female }\end{array}$ \\
\hline SK1 & 5 & 9 & 7 & 9 \\
\hline SK2 & 2 & 1 & 0 & 1 \\
\hline
\end{tabular}
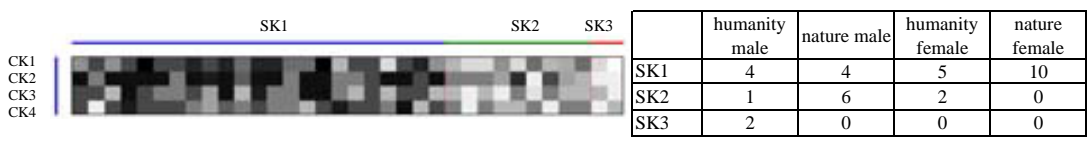

SK1

SK2 SK3

\begin{tabular}{|l|c|c|c|c|}
\hline & $\begin{array}{c}\text { humanity } \\
\text { male }\end{array}$ & nature male & $\begin{array}{c}\text { humanity } \\
\text { female }\end{array}$ & $\begin{array}{c}\text { nature } \\
\text { female }\end{array}$ \\
\hline SK1 & 4 & 9 & 7 & 10 \\
\hline SK2 & 2 & 1 & 0 & 0 \\
\hline SK3 & 1 & 0 & 0 & 0 \\
\hline
\end{tabular}

Figure 2. Influence of each feature cluster on the formation of the four concept clusters across 34 subjects

answering the feature association test so that the feature clusters are divided into the more specific clusters. On the other hand, the more specific concept clusters are identified by the group of male humanities than the group of male natural sciences. The explanation assumed from comments obtained from several subjects in male natural sciences is that they were simply not familiar with these clothing items expressed in English. Thus, their feature-association test is subject to more uncertainty. One interesting point is that the subject group in male humanities differently clusters the English word, shorts belonging to casual items $C K 1$, which is reasonable, because, in English, shorts is usually considered as 'short-legged trousers'. However, it seems that the other three groups of subjects associated shorts with 
'female underwear', probably because the English word shorts is expressed in Japanese as Katakana loan words and is widely used as synonym of panties in, e.g., Japanese department stores etc.. The male humanities who are familiar with native English communications probably distinguished this difference. This implies that English proficiency also seems to influence the conceptualization and categorization of domain knowledge expressed in English. For supporting this hypothesis, we selected three matrices generated by advanced English learners and conducted the multi-array IRM analysis in Fig. 1, which resulted in more fine-grained concept clusters with similar feature association patterns. The above interpretation requires further investigation, e.g., by contrasting with results obtained from the picture-naming test and the categorization test briefly explained in Section 2. Due to the limited space in this paper, we will not discuss the result of these tests here.

Although further investigations are needed, conceptualization patterns represented as feature associations by people having different backgrounds can, to a certain extent, be investigated by the presented approach. The approach presented in this paper focused on the multi-array analysis which in parallel bi-clusters two dimensional matrices. Our future perspective is to employ the multi-dimensional IRM method that simultaneously clusters three or multi dimensional matrices. The three dimensional IRM analysis would be able to automatically extract groups of subjects possessing similar concept-feature relations, from which subjects profiles constituting the extracted subject groups are investigated. Such approach would be useful not only for the analysis of semantic representations but also consumer behavior predictions where, e.g., products, product specifications and subjects are defined as three modes of a matrix.

Our ambition is to extend the analysis of semantic representations to global scale by conducting the same questionnaires in several countries, i.e. Denmark, China etc. This will hopefully lead to another ambitious project to realize crosscultural English communication simulation between people having different backgrounds described in [10].

\section{CONCLUSION}

In this paper, the semantic representations of 34 subjects with different profiles in gender and educational background are analyzed by the approach combining the multi-array IRM and the normal IRM. The employed approach identified similar patterns of conceptualization within a specific group of subjects as well as differences in conceptualization among individuals and groups of subjects. Although further investigation is required for the flawless interpretation, the obtained results indicated some phenomena specific to a subject group possessing the same background profile. The results also indicated that the employed models could be further extended to a three dimensional IRM for automatically extracting group of subjects possessing similar patterns of conceptualizations.

\section{ACKNOWLEDGMENT}

The survey conducted in this paper is supported by the Scandinavia-Japan Sasakawa Foundation. We would like to express our gratitude to Profs. Mark C. Hamilton and Eric S. Dean who supported the survey at Dept. of International Communication, Tokai University, Sapporo, Japan.

\section{REFERENCES}

[1] C. Kemp, J. B. Tenenbaum, T. L. Griffiths, T. Yamada, and N. Ueda, "Learning systems of concepts with an infinite relational model," in The Twenty-First National Conference on Artificial Intelligence, 2006.

[2] M. Mørup, K. H. Madsen, A. M. Dogonowski, H. Siebner, and L. K. Hansen, "Infinite relational modeling of functional connectivity in resting state fmri," in Proceedings of Neural Information Processing Systems, 2010.

[3] T. Herlau, M. Mørup, M. N. Schmidt, and L. K. Hansen, "Modelling dense relational data," in IEEE International Workshop on Machine Learning for Signal Processing (MLSP), Santander, Spain, 2012.

[4] P. Durst-Andersen, Linguistic Supertypes: A Cognitivesemiotic Theory of Human Communication. Berlin-New York: De Gruyter Mouton, 2011.

[5] G. L. Murphy, The Big Book of Concepts. Cambridge, Massachusetts: The MIT Press, 2004.

[6] F. K. Glückstad, T. Herlau, M. N. Schmidt, and M. Mørup, "Analysis of subjective conceptualizations towards collective conceptual modelling," in 27th Annual Conference of the Japanese Society for Artificial Intelligence, Japan, 2013.

[7] S. De Deyne, S. Verheyen, E. Ameel, W. Vanpaemel, M. Dry, W. Voorspoels, and G. Storms, "Exemplar by feature applicability matrices and other dutch normative data for semantic concepts," Behavior Research Methods, vol.40(4), pp. 10301048, 2008.

[8] J. Pitman, "Combinatorial stochastic processes," in Booktitles for Saint Flour Summer School, 2002.

[9] L. Danon, A. Díaz-Guilera, J. Duch, and A. Arenas, "Comparing community structure identification." Journal of Statistical Mechanics: Theory and Experiment, 2005.

[10] F. K. Glückstad, "Aligning mental representations," Journal of Advanced Computational Intelligence and Intelligent Informatics, Vol.17(4), pp. 663-669, 2013. 